\title{
Wip-ing out cancer
}

\author{
René Bernards
}

The Wip 1 phosphatase is encoded by an oncogene that is amplified in several forms of human cancer, including breast cancer. Ablation of this gene confers resistance to breast tumors induced by certain oncogenes.

Cancer often results from a combination of activating mutations in growth-stimulatory genes (oncogenes) and inactivating mutations in growth-inhibitory genes (tumor-suppressor genes). Ideally, anticancer drugs should specifically inhibit the activity of the products of the oncogenes or reactivate the antiproliferative effects of the tumor-suppressor proteins. Such drugs would be more cancer-specific and have fewer side effects than the current generation of broad-specificity cytotoxic drugs. In spite of the difficulties in developing such designer drugs, the first successes have recently been reported ${ }^{1,2}$ and some have already reached the clinic with remarkable success. But which oncogene products are the best targets for the development of new anticancer drugs? On page 343-350 of this issue, Dmitry Bulavin and colleagues $^{3}$ provide compelling evidence that inhibiting Wip1 may be a good strategy for treating certain types of cancer.

\section{A brief history of Wip1}

Wip1 is a serine-threonine phosphatase encoded by Ppm1d. It was first identified as a gene that is induced by the p53 tumor-suppressor protein in response to DNA damage 4 . The importance of the activation of this phosphatase by p53 became clear when it was found that Wip1 specifically inactivates the protein kinase $\mathrm{p} 38 \mathrm{MAPK}$, which can activate p53 to cause cell cycle arrest and apoptosis in response to certain environmental stresses ${ }^{5}$. In addition, p38 MAPK can inhibit cell cycle stimulatory proteins, such as cyclin D1 and the Cdc25 phosphatases ${ }^{6,7}$. Thus, Ppm1d activation by $\mathrm{p} 53$ seems to constitute a negative

René Bernards is in the Division of Molecular Carcinogenesis and Center for Biomedical

Genetics, The Netherlands Cancer Institute, Amsterdam.

e-mail: r.bernards@nki.nl

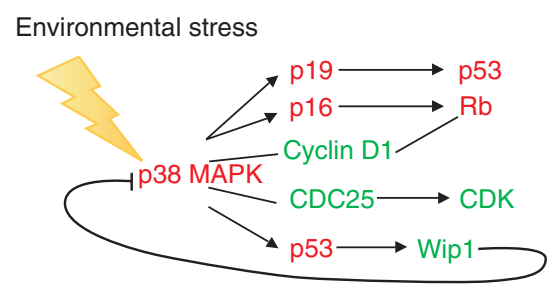

Figure 1 The role of the Wip1 phosphatase in the response to stress signaling. Environmental stresses, such as ultraviolet radiation, activate the p38 MAPK protein kinase. This leads to the activation of a number of growth inhibitory proteins (indicated in red) and inactivation of growthstimulatory proteins (green). The p53 tumorsuppressor protein activates several genes, including one that encodes the Wip1 phosphatase triggering a feedback inhibition loop to p38 MAPK.

feedback loop responsible for downregulation of genotoxic stress-induced signaling, which leads to suppression of the cellular stress response (Fig. 1).

As the p38 MAPK pathway is involved in negative regulation of cell proliferation, it is probably not surprising that overexpression of its inhibitor, Wip1, is seen in several forms of human cancer, including breast cancer ${ }^{8-10}$. Also consistent with a role for Wip1 in cell proliferation is the finding that Ppmld can cooperate with an activated Hras oncogene in transformation of primary mouse embryo fibroblasts $^{11}$ (MEFs). Conversely, Wip1-null MEFs have a reduced proliferative capacity in vitro and suffer from premature onset of senescence $^{12}$. Suppression of Ppmld expression in neuroblastoma cell lines suppressed growth and induced apoptosis ${ }^{8}$. Together, these data establish Wip1 as a central component of the cellular stress response, whose inhibition enhances antiproliferative effects of environmental stress signals.

\section{Multiple Wip1 targets}

In the present study, Bulavin et al. ${ }^{3}$ studied the effects of loss of the Wip1 phosphatase in vitro and in vivo. They used fibroblasts derived from Wip1-null mouse embryos, which were previously shown to have a very limited in vitro lifespan due to premature onset of senescence. Consistent with the idea that Wip1 is required for negative regulation of p53 in response to certain stress signals, Ppm1 $1 d^{-/-}$MEFs express elevated levels of the p53 target p21 (also called cip1; ref. 12). In spite of this, introduction of pairs of collaborating oncogenes, such as adenovirus E1A and Hras, restored normal proliferation rates in the Wip1-null cells, at least initially. But these in vitro-transformed cells were very poorly tumorigenic in nude mice. Subsequent analyses indicated that the transformed Wip1-null MEFs not only had elevated activity of p53 and its downstream targets, but also expressed higher levels of two additional tumor suppressors, p19 (also called Arf, an upstream regulator of p53) and p16 (also called Ink4a, an inhibitor of the cyclin DCDK4-CDK6 protein kinases, which in turn are upstream regulators of the retinoblastoma $(\mathrm{Rb})$ tumor-suppressor protein). Thus, deletion of Ppmld activated two distinct tumor suppressors: the $\mathrm{p} 53$ and $\mathrm{Rb}$ pathways.

Which of these two pathways is more important in protecting Wip1-null cells from tumorigenesis? In a series of genetic experiments, the authors dissected the contribution of these two pathways to the antioncogenic effects of Wip1 loss. First, they generated MEFs deficient in both Wip1 and p53. Even though these Wip1p53 double-knockout MEFs escaped the premature senescence response of the Wip1-null cells, they were still nontumorigenic when transformed with oncogenes, indicating that other antioncogenic pathways contribute to the transformation resistance of Wip1-null 
cells. In contrast, MEFs that lacked Wip1, p19 and p16 were fully oncogenic when the oncogenes Hras or Myc were introduced. MEFs that lacked only Wip 1 and p16 had an intermediate phenotype, as they were fully oncogenic when transformed by certain combinations of oncogenes, but not by others. The conclusion was that loss of Wip1 activates two tumor suppressor pathways, 19 and p16, both of which contribute to the resistance to transformation of cells lacking Wip1.

\section{Living proof}

Encouraged by the in vitro data, Bulavin et al. ${ }^{3}$ went on to ask if ablation of Ppmld would inhibit oncogenesis in vivo. The authors decided to study breast cancer development, as Wip1-null mice have a strong induction of p16 in the normal mammary epithelium, and previous work indicated that the presence of cyclin D1 (whose action is inhibited by p16) is required for induction of breast cancer by certain oncogenes ${ }^{13}$. The authors crossed mice deficient for Wip1 with three different strains of mice, each engineered to overexpress a different oncogene in the epithelium of the mammary gland. Bulavin et al. ${ }^{3}$ found that mammary tumorigenesis in mice that expressed the Wnt1 oncogene in the mammary gland was not affected by the absence of Wip1, whereas mice that expressed either Hras or Erbb2 in the breast epithelium were relatively resistant to the development of breast cancer in the absence of Wipl. Finally, the authors show that when treated with a specific p38 MAPK inhibitor, tumor-resistant Wip1null mice that express Erbb2 in the breast repressed expression of p16 and developed breast tumors. Together, these data indicate that the absence of Wip1 prevents breast cancer induction through constitutive activation of p38 MAPK, which in turn causes upregulation of the tumor suppressor p16 (Fig. 1).

\section{Wip1 as a drug target}

The present study indicates that inhibition of the Wip1 phosphatase could suppress the proliferation of certain types of cancer, most notably breast cancer. Phosphatases are, in principle, susceptible to targeting by drugs, as potent inhibitors of other phosphatases have been developed. The side effects of inhibition of Wip1 may also be acceptable, as Wip1-null mice develop normally, even though defects in immune function have been noted ${ }^{12}$. Not all types of cancer respond to antiproliferative signaling through the 16 or p19 tumor-suppressor pathways. Indeed, Bulavin et al. ${ }^{3}$ show that breast cancers caused by the Wnt 1 oncogene in mice are not inhibited by loss of Wip1. But a substantial fraction of breast cancers have increased expression of cyclin D1, and such tumors may benefit from Wip1 inhibition ${ }^{14}$.

1. Lane, D.P. \& Fischer, P.M. Nature 427, 789-790 (2004).

2. Druker, B.J. Cancer Cell 1, 31-36 (2002).

3. Bulavin, D.V. et al. Nat. Genet. 36, 343-350 (2004).

4. Fiscella, M. et al. Proc. Natl. Acad. Sci. USA 94, 6048-6053 (1997).

5. Takekawa, M. et al. EMBO J. 19, 6517-6526 (2000).

6. Lavoie, J.N., L'Allemain, G., Brunet, A., Muller, R. \& Pouyssegur, J. J. Biol. Chem. 271, 20608-20616 (1996).

7. Bulavin, D.V. et al. Nature 411, 102-107 (2001).

8. Saito-Ohara, F. et al. Cancer Res. 63, 1876-1883 (2003).

9. Li, J. et al. Nat. Genet. 31, 133-134 (2002).

10. Hirasawa, A. et al. Clin. Cancer Res. 9, 1995-2004 (2003).

11. Bulavin, D.V. et al. Nat. Genet. 31, 210-215 (2002).

12. Choi, J. et al. Mol. Cell. Biol. 22, 1094-1105 (2002).

13. Yu, Q., Geng, Y. \& Sicinski, P. Nature 411, 1017-1021 (2001).

14. van Diest, P.J. et al. Am. J. Pathol. 150, 705-711 (1997).

\title{
Shutting down Wnt signal-activated cancer
}

\author{
M Mark Taketo
}

\section{New evidence suggests that Wnt signaling can be suppressed or further activated by upstream signals, even though the pathway seems to be constitutively activated by downstream mutations in cancer cells.}

The Wnt signal pathways have key roles in embryonic development. Defects in the pathway have also been implicated in cancer of the colon and other organs ${ }^{1,2}$. Two Wnt pathways have been identified: the canonical and noncanonical pathways. Activation of the canonical pathway induces transcription of a new set of genes through the $\beta$ catenin-T cell factor (TCF) complex, which regulates cell proliferation and differentiation $^{1}$. Activation of the noncanonical pathway does not require $\beta$-catenin signaling and controls cell movement during morphogenesis ${ }^{2}$.

In the absence of Wnt ligands bound to their receptors, the cytoplasmic complex of APC and Axin provide a scaffold for GSK3 $\beta$

M. Mark Taketo is in the Department of Pharmacology, Kyoto University School of Medicine, Kyoto, Japan.

e-mail: taketo@mfour.med.kyoto-u.ac.jp to phosphorylate $\beta$-catenin (Fig. 1a). Phosphorylated $\beta$-catenin is then rapidly degraded through the ubiquitin pathway. When Wnt ligands bind to the cell-surface receptor Frizzled (Fzd), they trigger the phosphorylation of a cytoplasmic effector, Dishevelled (Dsh), which then inhibits the activity of GSK3 $\beta$ on the APC-Axin complex. Unphosphorylated, and therefore stable, $\beta$-catenin can then accumulate in the cytoplasm and form a complex with TCF in the nucleus, which initiates transcription of Wnt target genes (Fig. 1b).

\section{Canonical Wnt signaling in cancer}

Most colon cancers and other digestive cancers are associated with mutations in APC, AXIN1 or CTNNB1, and $\sim 90 \%$ of colon cancers are associated with defects in the canonical Wnt signaling pathway (Fig. 1c) ${ }^{1}$. Mutant APC and Axin are unable to assist GSK3 $\beta$ in phosphorylating $\beta$-catenin. Similarly, mutations that lead to amino acid substitutions in the phosphorylated residues of $\beta$-catenin stabilize the protein. Either type of disruption causes constitutive signaling independent of the upstream signal from Wnt.

On page 417-422, Hiromu Suzuki and colleagues add a new twist to this simplistic view on the canonical Wnt pathway ${ }^{3}$. In an earlier paper, they isolated genes that were preferentially hypermethylated in human colon and gastric cancers ${ }^{4}$. Among them, they identified a family of secreted Fzdrelated proteins (SFRPs) that can compete with Fzd for the Wnt ligands. Now, the authors report on experiments in which they expressed SFRPs in colon cancer cell lines carrying mutations in CTNNB1 or APC. SFRP1, SFRP2 and SFRP5 suppressed Wntdependent transcription by $\sim 60 \%$ (Fig. 1d). They then expressed WNT1 in the $\beta$-catenin mutant cell line HCT116. Wnt pathwaydependent transcription was $\sim 3$ times 